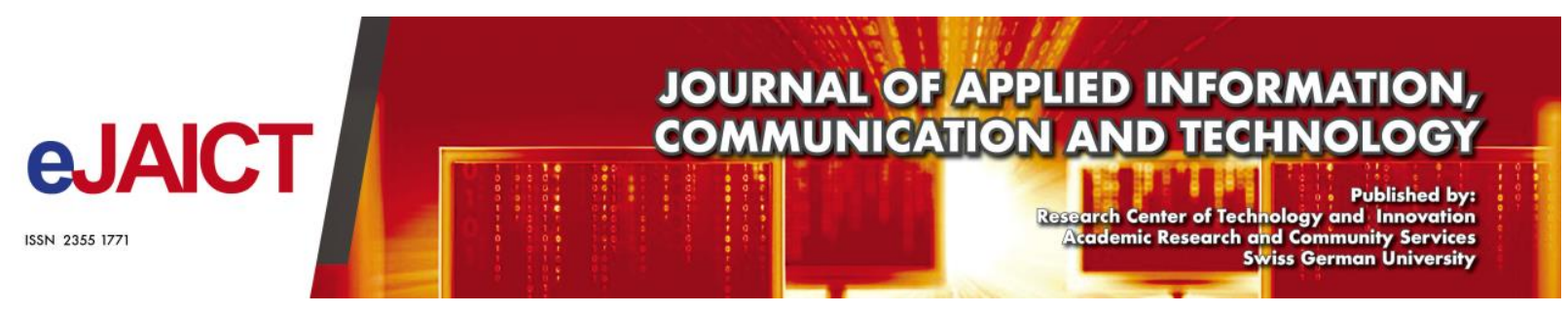

\title{
Pendulum with Aerodynamic and Viscous Damping
}

\section{Herlin Soraya}

Faculty of Engineering and Information Technology, Swiss German University

\section{Article Information}

Received: 20 June 2016

Accepted: 29 August 2016

Published: 25 October 2016

DOI: $10.33555 /$ ejaict.v3i2.94

Corresponding Author:

Herlin Soraya

Email:

ISSN 2355-1771

\begin{abstract}
In this paper we discuss about how the relationship between non-linear differential equations on aerodynamic damping with linearly viscous damping equations. And it turns out after analyzing that the changes that occur pendulum that changes from the start of the maximum state to a stable state takes time so that changes that occur until the state is stable, this change can be reduced with the use of viscous damper.
\end{abstract}




\section{Introduction and Theory}

In this theory we assume that the oscillation system movement is a pendulum with a compound image consisting of an oscillation shape so that the change can be calculated theoretical and measured. The system can be muffled by immersing the free pendulum tip in viscous fluid. Observations were made with the natural frequency of the pendulum that was not submerged, the pendulum behavior with attenuation. Movement as a function of time is recorder directly by fixed the pen to the pendulum and let it write on the moving chart.

Following a picture of a pendulum compound like Figure 1 consisting of heavy rods that have large discs attached to it. We show the moment of inertia in the pivot to the center of gravity is L, and the length of the trunk is 1 .

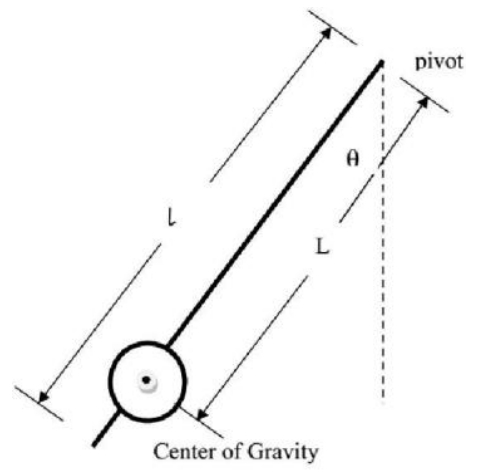

Figure 1.

As we know that a pendulum will experience a simple harmonic. There will be a movement when there is an external force working on the pendulum and the pendulum is experiencing The angular displacement is modeled as a time function, assuming the pendulum maintains the angular amplitude and initiates Oscillation at the angular amplitude as show in figure 1.

To solve this differential equation is needed to be first order differential.

$8=y(1)$
$8^{\prime}=y(2)$

Both equation become:

$\mathrm{y}^{\prime}(1)=\mathrm{y}(2)$

$\mathrm{y}^{\prime}(2)=\underset{\mathrm{S}}{(-\mathrm{g})} *(\mathrm{y}(1))-\underset{\mathrm{N}}{\left(\frac{\mathrm{Cd}}{)}\right)} *(\mathrm{y}(2))$

Similar equation become:

$y^{\prime}(1)=y(2)$

$y^{\prime}(2)=(-g) * \sin (y(1))-\left(\frac{C d}{S}\right) *(y(2) * 2) \operatorname{sgn}(y(2))$

To solve these two problems with aerodynamic drum pendulum and pendulum with viscous damping attenuation. so we have to write in 2 files [pendulum1.m] and 
[pendulum2.m] which are derived from this problem with aerodynamic drag and derivation of the problem with viscous damping attenuation.

Here is the code that must be entered into the MATLAB program containing the equations that have been created from equations 5 and 6 .

Note the existing function di Matlab by improving some signs that do not work with marks that exist in Matlab function:

yp $=$ pendulum $1(t, y)$

$\mathrm{m}=10$

$\mathrm{g}=981$

$1=5$;

$\mathrm{Ca}=14$

$y p=\left[y(2) ;\left((-g / 1) * \sin (y(1))-C a /(m * 1) *\left(y(2)^{\wedge} 2\right)^{*} \operatorname{sign}(y(2))\right)\right] ;$

function $y p=$ pendulum $2(t, y)$

$\mathrm{m}=10$

$\mathrm{g}=981$

$1=5$;

$\mathrm{Ca}=14$

$\mathrm{yp}=[\mathrm{y}(2) ;((-\mathrm{g} / \mathrm{l}) *(\mathrm{y}(1))-(\mathrm{Ca} / \mathrm{m}) *(\mathrm{y}(2)))]$

\subsection{Pendulum Main}

$\operatorname{tspan}=\left[\begin{array}{ll}0 & 10\end{array}\right]$

$\mathrm{y} 0=[1.57 ; 0]$

$[\mathrm{t}, \mathrm{y}]=$ ode45('pendulum 1',tspan,y0);

$\operatorname{plot}(\mathrm{t}, \mathrm{y}(:, 1)) ;$ grid

xlabel('Time')

ylabel('Theta')

title('Theta Vs Time') 
hold on;

$\operatorname{tspan}=\left[\begin{array}{ll}0 & 10\end{array}\right] ;$

$\mathrm{y} 0=[1.57 ; 0]$

$[\mathrm{t}, \mathrm{y}]=$ ode45('pendulum2',tspan,y0);

$\operatorname{plot}(\mathrm{t}, \mathrm{y}(:, 1)) ;$ grid

xlabel('Time'

ylabel('Theta')

title('Theta Vs Time')

hold on;

\subsection{Pendulum Mains}

$\operatorname{tspan}=\left[\begin{array}{ll}0 & 10\end{array}\right]$

$\mathrm{y} 0=[1.57 ; 0]$

$[\mathrm{t}, \mathrm{y}]=$ ode45('pendulum2',tspan,y0);

$\operatorname{plot}(\mathrm{t}, \mathrm{y}(:, 1))$;grid

xlabel('Time'

ylabel('Theta')

title('Theta Vs Time')

hold on;

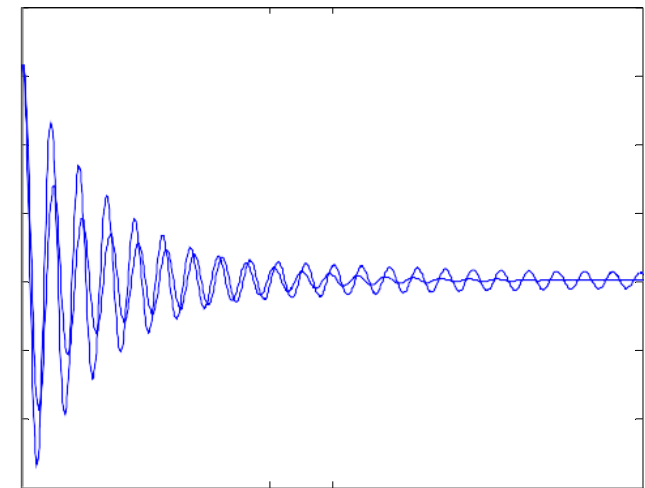

Figure 2. Comparison between Quadratic and Linier Damping 


\section{Conclusion}

In this study shown that typical pendulum dynamics is far from the simple equation of motion a reasonably complete damping model shall use nonlinear terms in addition to the common linear viscous expression. more appropriate in some cases a nonlinear substitute for assumed linear damping. Even though some of exceptional cases where all nonlinearity may be ignored, it is shown that viscous damping involves subtleties that can lead errors when it is ignored.

\section{References}

Modeling Damping for a pendulum. Sherman IP: A Department of Physics and Astronomy, University College London.

Nonlinear Damping of the 'Linear'. Department of Physics. Macon, Georgia: Mercer University.

Palm, W.J. System Dynamics. University of Rhode Island.

Syahriar, A. Modelling and Simulation of Mechanical System, Lecture 1\#.

Syahriar, A. Modelling of Rigid-Body: Mechanical Systems, Lecture 3\#.

The Damped Motion of a Compound Pendulum. Physical Department, Brown University. 